\title{
Other drug treatments: the evidence
}

\author{
Neil Poulter \\ Cardiovascular Studies Unit, Department of Clinical Pharmacology, Imperial College School of Medicine, \\ St Mary's Campus, London W2 1PG, UK
}

Robust data are available to support the use of several drug classes for the prevention of cardiovascular disease. The field of hypertension, particularly, has more large randomized controlled trials evaluating the impact of interventions on morbidity and mortality than almost any other area of medicine.

The combined results of most of these trials, which included about 47000 hypertensive patients, revealed that reduction of $5-6 \mathrm{mmHg}$ in diastolic blood pressure using diuretic and/or $\beta$-blocker-based regimens was associated with about a $38 \%$ reduction in stroke risk and about a $16 \%$ reduction in CHD risk (Collins et al. 1990).

More recently, evidence regarding the effects of the 'newer' classes of blood pressure-lowering agents (Ca-channel blockers, and angiotensin converting enzyme (ACE) inhibitors) on morbidity and mortality have been published.

Subgroup analyses of some of these trials have allowed an evaluation of the effects of various drugs in specific subgroups of patients, and other trials have targeted specific subgroups of patients which will provide guidance on the advisability or otherwise of tailoring anti-hypertensive therapy.

Evidence from Hypertension Optimal Treatment (HOT) and UK Prospective Diabetes Study Group (UKPDS) trials have reinforced prospective epidemiological data that targets for blood pressure reduction should be reduced, at least in subgroups.

Until recently, the field of diabetes had virtually no good trial evidence to guide practice regarding the prevention of macrovascular cardiovascular disease. However, more recently, the UKPDS trial has been published to make a major contribution to available data in the area. A key message to arise from UKPDS is that tight glycaemic control achieved by oral hypoglycaemic agents and insulin did reduce microvascular complications compared with less-tight control, and the data also suggested that macrovascular complications were reduced with tight control. It is worth noting that tight blood pressure control compared with less-tight blood pressure control was more effective than glycaemic control with regard to the prevention of macrovascular events.

The use of anti-platelet agents (mainly aspirin) is clearly established in the context of secondary prevention. Use in primary prevention is more contentious where risk: benefit has probably to be judged carefully before aspirin use is recommended. In general, low-dose aspirin is probably appropriate for those subjects over the age of 50 years who have their blood pressure controlled and in whom estimated cardiovascular disease risk is $\geq 2 \%$ per annum (Ramsey et al. 1999).

A series of randomized controlled trials have clearly established the use of ACE inhibitors for those with heart failure and impaired left ventricular systolic function after myocardial infarction. Latterly trials of $\beta$-blockers have also shown great benefits for patients in heart failure. Whether the more-recently-introduced angiotensin II antagonists will prove to be equally effective or more effective than ACE inhibitors for such patients is currently being validated in trials.

The use of nicotine replacement therapy with patches, gum and inhalators has been shown in trials to approximately double smoking cessation rates irrespective of adjunctive support. The use of such products should therefore be increasingly encouraged.

Although popular, the use of antioxidants for the prevention of cardiovascular disease is not as yet established on the basis of trial evidence. Indeed, although one trial has shown reduced coronary morbidity associated with the use of vitamin $E$ after myocardial infarction, the impact of antioxidants on mortality in several trials has been at best worrisome.

On the basis of observational data the use of folic acid supplements to lower blood homocysteine levels may be of benefit in relation to several types of cardiovascular events. However, as yet, no trial evidence exists to support the use of folic acid for this purpose, although trials are in progress.

\footnotetext{
Abbreviations: ACE, angiotensin converting enzyme; HOT, Hypertension Optimal Treatment; UKPDS, UK Prospective Diabetes Study Group. Corresponding author: Professor Neil Poulter, fax +44 (0) 207594 3411, email sandra.johnson@ic.ac.uk
} 
In summary, therefore, we do have extensive evidence for the benefits of several types of agents for preventing cardiovascular disease particularly anti-hypertensive agents, aspirin in secondary prevention, and ACE inhibitors and $\beta$-blockers for heart failure. Unfortunately, surveys of the use of such agents repeatedly confirm that the evidence supporting the use of these agents is all too frequently ignored (Colhoun et al. 1998).

\section{Cardiovascular disease: Drug treatments: Anti-hypertensive therapy}

\section{References}

Colhoun HM, Dong W \& Poulter NR (1998) Blood pressure screening, management and control in England; results from the Health Survey for England 1994. Journal of Hypertension 16, 747-753.

Collins R, Peto R, Macmahon S, Hebert P, Fiebach NH, Eberlein KA, Godwin J, Qizilbash N, Taylor JO \& Hennekens CH (1990) Blood pressure, stroke, and coronary heart disease. Part 2, short-term reductions in blood pressure: overview of randomised drug trials in their epidemiological context. Lancet 335, 827-838.

Ramsay LE, Williams B, Johnston GD, MacGregor GA, Poston L, Potter JF, Poulter NR \& Russell G (1999) Guidelines for management of hypertension: Report of the third working party of the British Hypertension Society, 1999. Journal of Human Hypertension 13, 569-592. 